\title{
Model Study and Fault Detection for the Railway System
}

\author{
ARFA Rawia \\ Department of Electrical \\ Engineering, University of Tunis \\ El Manar, LA.R.A. Automatique, \\ National Engineering School of \\ Tunis, BP 37, 1002 Tunis, TUNISIA
}

\author{
TLIJANI Hatem \\ Department of Electrical \\ Engineering, University of Tunis \\ El Manar, LA.R.A. Automatique, \\ National Engineering School of \\ Tunis, BP 37, 1002 Tunis, TUNISIA
}

\author{
KNANI Jilani \\ Department of Electrical \\ Engineering, University of Tunis \\ El Manar, LA.R.A. Automatique, \\ National Engineering School of \\ Tunis, BP 37, 1002 Tunis, TUNISIA
}

\begin{abstract}
The wheel-rail-sleepers system is simulated as a series of moving point loads on an Euler-Bernoulli beam resting on a visco-elastic half space. This paper concentrates on the railsleepers interaction system (railway system) and the fault detection. The main objective is to mathematically develop and implement a dynamic model of a railway system then the diagnosis of system defects using a Luenberger observer (LO). The simulation results are based on a physical description, mathematical equations and simulations with MATLAB simulation program.
\end{abstract}

Keywords-Dynamic model; the wheel-rail-sleepers system; interaction system; Euler-Bernoulli; Luenberger observer (LO); fault detection

\section{INTRODUCTION}

Because of the importance of the safety and the great railsleepers interaction effect on it, in the last few years there has been a growing interest in the diagnosis of the railway system and the rail-sleepers interaction induced by moving high-speed trains. The early detection and isolation are very important to avoid costly breakdowns and improve equipment reliability as reported in [1] and [2]. In the past decades, various analytical, semi-analytical and numerical methods with different accuracies have been attracted much attention from research teams to investigate the rail vibrations then the diagnosis of this system and isolation of the defect [23], [24], [25] and [26]. The finite elements method [29] is a very effective method for the railway model calculates. This paper is focused on this method for the model calculation.

There are essentially two opposing attitudes for diagnosis system [22] and [27]. First one is corrective maintenance or diagnosis after the failure, second one is the preventive maintenance [28] or diagnosis before the failure. While the second attitude seems more attractive, it's not systematically applied on industrial process. In practice, these two approaches still coexist. Previous studies indicate that the good maintenance is to implement the most appropriate technique for each device, sub-assembly or each element. The maintenance type choice is based on the knowledge available on the installation and objectives.

To diagnose and isolate the fault of the railway system, it must first calculate its dynamic model [30] and [31]. The model considers the rail and the sleepers as a whole system and couples the vertical interaction with the lateral interaction. To calculate the railway model it must consider the wheel-rail interaction. The vertical contact forces between wheels and rails are described by Hertzian nonlinear elastic contact theory and the tangential wheel-rail forces are decided by the creep theory. Generally, there are three main classes of dynamic interactions between rails and sleepers: vertical, lateral and torsional. Vertical rail-sleepers interactions are responsible for damage and service life of the system [3] demonstrated that. Lateral and torsional interactions usually influence the safety against derailment of train and wear of wheels and rails. In fact, the vertical and lateral rail-sleepers interactions can't be separated from each other.

So this work is a mathematical calculation of the railway model, then the isolation of default with Luenberger Observer (LO) [21].

Section II describes the real system in which this work is applied. Section III analyzes the system model where the dynamic model of the system is calculated. Section IV the different variation cases of the system where there is the global model study of the railway system. Section $\mathrm{V}$ concentrates for the fault detection where there is study of observer type used in this work. Choice of Luenberger observer for the diagnosis and fault isolation, calculation of parameters and experimental results are presented in Section VI; Section VII concludes the paper.

\section{SySTEM DESCRIPTION}

The system is a train rail. The rail treated as a rigid model in which it is supported on two double-axles with the primary and the secondary suspension systems. The primary suspensions, connecting the wheels, are represented by ordinary Kelvin elements. The secondary suspensions, connecting the sleepers, are also modeled as ordinary Kelvin elements.

The wheels and sleepers are coupled through the suspension elements. The rail and sleepers each undergo lateral displacement and vertical, but only vertical displacement is considered in the four wheels. More knowledge about this system presented in [4]-[6]. 
The dynamic model of railway system can be presented in the form of equations of motions vertical, lateral and torsional [7].

\section{MATHEMATIC MODEL}

There are several experiments in order to verify the validity of the infinite element method [8]-[15].

The research in this work started by these equations that describe the motion of the rail is as follows:

- Vertical Motion:

$$
\begin{aligned}
& E I_{y} \frac{d^{4} Z_{r}(x, t)}{d x^{4}}+m_{r} \frac{d^{2} Z_{r}(x, t)}{d t^{2}} \\
& =-\sum_{i=1}^{N} F_{v i}(t) \delta\left(x-x_{i}\right)+\sum_{j=1}^{4} G_{v j}(t) \delta\left(x-x_{g}\right)
\end{aligned}
$$

- Lateral Motion:

$$
\begin{aligned}
& E I_{z} \frac{d^{4} Y_{r}(x, t)}{d x^{4}}+m_{r} \frac{d^{2} Y_{r}(x, t)}{d t^{2}} \\
& =-\sum_{i=1}^{N} F_{L i}(t) \delta\left(x-x_{i}\right)+\sum_{j=1}^{4} G_{L j}(t) \delta\left(x-x_{g}\right)
\end{aligned}
$$

- Torsional Motion:

$$
\begin{aligned}
& \rho I_{0} \frac{d^{2} \phi_{r}(x, t)}{d t^{2}}-G K \frac{d^{2} \phi_{r}(x, t)}{d x^{2}} \\
& =-\sum_{i=1}^{N} M_{F i}(t) \delta\left(x-x_{i}\right)+\sum_{j=1}^{4} M_{G j}(t) \delta\left(x-x_{g}\right)
\end{aligned}
$$

Where $Z_{r}(x, t), \mathrm{Y}_{r}(x, t)$ and $\phi_{r}(x, t)$ are respectively the vertical, lateral and torsional displacements of de rail, EIy and EIz are the rail bending stiffness to the $y$-axle and to the $z$ axels respectively, $\mathrm{mr}$ the rail mass per unit length, $\rho$ the rail density, $\mathrm{I}_{0}$ the torsional inertia of rail, GK the rail torsional stiffness and $\delta(x)$ the Dirac delta function.

Equation (4) represents the vertical and lateral dynamic forces of the i-th rail/sleeper point.

$$
\left\{\begin{array}{l}
F_{v i}(t)=K_{p v}\left(Z_{r}\left(x_{i}, t\right)-Z_{s i}(t)-a \phi_{s i}(t)\right)+C_{p v}\left(\dot{Z}_{r}\left(x_{i}, t\right)-\dot{Z}_{s i}(t)-a \dot{\phi}_{s i}(t)\right) \\
F_{L i}(t)=K_{p h}\left(Y_{r}\left(x_{i}, t\right)-Y_{s i}(t)-a \phi_{s i}(t)\right)+C_{p h}\left(\dot{Y}_{s i}(t)-a \dot{Y}_{s i}(t)\right)
\end{array}\right.
$$

Where, $\mathrm{Z}_{\mathrm{si}}(\mathrm{t}), \mathrm{Y}_{\mathrm{si}}(\mathrm{t})$ and $\phi_{s i}(t)$ Vertical, lateral and torsional displacements of the i-th sleepers They can be assumed to be zero, $\mathrm{K}_{\mathrm{pv}}$ and $\mathrm{K}_{\mathrm{ph}}$ are the vertical and lateral stiffness between the rail and the sleepers and $\mathrm{C}_{\mathrm{pv}}$ et $\mathrm{C}_{\mathrm{ph}}$ are the vertical and lateral rail/sleeper damping.

The dynamic of wheel/rail forces can be written as (5), where $G_{v j}(t)$ vertical forces and $G_{L J}(t)$ lateral forces.

$\left\{\begin{array}{l}G_{v j}(t)=P_{j}(t)-P_{0} \\ G_{L j}(t)=Q_{j}(t)\end{array}\right.$
$\mathrm{P}_{\mathrm{j}}(\mathrm{t})$ and $\mathrm{Q}_{\mathrm{j}}(\mathrm{t})$ are the $\mathrm{j}$-th wheel/rail forces and $\mathrm{P}_{0}$ the static wheel load.

Equation (6) defines the moments of forces acting on the rail due to fastening system and due to wheel / rail forces.

$$
\left\{\begin{array}{l}
M_{f i}(t)=K_{\psi} \phi_{r}\left(x_{i}, t\right) \\
M_{g j}(t)=G_{L j}(t) e_{1}-G_{v j}(t) e_{2}
\end{array}\right.
$$

Where, $K_{\psi}$ the torsional stiffness of the fastening system, $\mathrm{e}_{1}$ and $e_{2}$ are the arms of the lateral and vertical wheel/rail forces to the torsional canter of the rail.

To facilitate the solution of motion differential equations of the rail, the following railway mode shape functions are assumed:

- Vertical Vibration

$$
Z_{k}(x)=\sqrt{\frac{2}{m_{r} l}} \sin \left(\frac{k \pi x}{l}\right)
$$

- Lateral Vibration

$$
\mathrm{Y}_{k}(x)=\sqrt{\frac{2}{m_{r} l}} \sin \left(\frac{k \pi x}{l}\right)
$$

- Torsional Vibration

$$
\phi_{k}(x)=\sqrt{\frac{2}{\rho I_{0} l}} \sin \left(\frac{k \pi x}{l}\right)
$$

Where 1 is the length of the rail and $\mathrm{k}$ is the mode number.

Thus, the characteristic variables of (1), (2) and (3) can be expressed as:

$$
\begin{aligned}
& Z_{r}(x, t)=\sum_{k=1}^{K} Z_{k}(x) q_{z k}(t) \\
& Y_{r}(x, t)=\sum_{k=1}^{K} Y_{k}(x) q_{y k}(t) \\
& \phi_{r}(x, t)=\sum_{k=1}^{K} \phi_{k}(x) q_{t k}(t)
\end{aligned}
$$

$q_{z k}(t), q_{y k}(t)$ and $q_{t k}(t)$ are the k-th mode time coordinate and $\mathrm{K}$ is the total number of modes considered.

Then the second order ordinary differential equations simplified in terms of the time coordinates (1), (2) and (3) can be computed by the following equation:

- Vertical Motion

$$
\begin{gathered}
\ddot{q_{z k}}(t)+\frac{E I_{y}}{m_{r}}\left(\frac{k \pi}{l}\right)^{4} q_{z k}(t)=-\sum_{i=1}^{N} F_{v i}(t) Z_{k}\left(x_{i}\right)+\sum_{j=1}^{4} G_{v j}(t) Z_{k}\left(x_{g i}\right) \\
K=1,2, \ldots, k
\end{gathered}
$$

- Lateral Motion 


$$
\begin{gathered}
\ddot{q_{y k}}(t)+\frac{E I_{z}}{m_{r}}\left(\frac{k \pi}{l}\right)^{4} q_{y k}(t)=-\sum_{i=1}^{N} F_{L i}(t) Y_{k}\left(x_{i}\right)+\sum_{j=1}^{4} G_{L j}(t) Y_{k}\left(x_{g i}\right) \\
K=1,2, \ldots, k
\end{gathered}
$$

- Torsional Motion

$$
\begin{gathered}
\ddot{q_{t k}}(t)+\frac{G K}{\rho I_{0}}\left(\frac{k \pi}{l}\right)^{4} q_{t k}(t)=-\sum_{i=1}^{N} M_{f i}(t) \phi_{k}\left(x_{i}\right)+\sum_{j=1}^{4} M_{g j}(t) \phi_{k}\left(x_{g i}\right) \\
K=1,2, \ldots, k
\end{gathered}
$$

If $\mathrm{K}$ fixed by the value 1 and with a small change of the variables the vertical, lateral and torsional vibration system of the rail could be mathematically written as:

- Vertical movement equation

$M_{z} \ddot{q_{z 1}}(t)+C_{z} \dot{q_{z 1}}(t)+K_{z} q_{z 1}(t)+C_{z y} \ddot{q_{t 1}}(t)+K_{z y} q_{t 1}(t)=P_{z}(t)$

Where

$$
\begin{aligned}
& M_{z}=1, C_{z}=\sum_{i=1}^{N} C_{p v}\left(\left(Z_{1}\left(x_{i}\right)-Z_{1}\left(x_{s i}\right)\right) Z_{1}\left(x_{i}\right)\right) \\
& C_{z y}=-a C_{p v} \sum_{i=1}^{N}\left(\phi_{1}\left(x_{s i}\right) Z_{1}\left(x_{i}\right)\right) \\
& K_{z}=\sum_{i=1}^{N}\left(K_{p v}\left(Z_{1}\left(x_{i}\right)-Z_{1}\left(x_{s i}\right)\right) Z_{1}\left(x_{i}\right)\right)+\frac{E I_{y}}{m_{r}}\left(\frac{k \pi}{l}\right)^{4}, \\
& K_{z y}=-a K_{p v} \sum_{i=1}^{N}\left(\phi_{1}\left(x_{s i}\right) q_{t 1}(t) Z_{1}\left(x_{i}\right)\right) \\
& \text { and } P_{z}(t)=\sum_{j=1}^{4}\left(P_{j}(t)-P_{0}\right) Z_{1}\left(x_{g i}\right)
\end{aligned}
$$

- Lateral movement equation

$$
M_{y} \ddot{q_{y 1}}(t)+C_{y} \dot{q_{y 1}}(t)+K_{y} q_{y 1}(t)=P_{y}(t)
$$

Where $M_{y}=1, C_{y}=C_{p h} \sum_{i=1}^{N}\left(Y_{1}\left(x_{i}\right)-Y_{1}\left(x_{s i}\right)\right) Y_{1}\left(x_{i}\right) \quad$,

$K_{y}=\frac{E I_{z}}{m_{r}}\left(\frac{k \pi}{l}\right)^{4}+K_{p h} \sum_{i=1}^{N}\left(\left(Y_{1}\left(x_{i}\right)-Y_{1}\left(x_{s i}\right)\right) Y_{1}\left(x_{i}\right)\right.$

$P_{y}(t)=\sum_{j=1}^{4} G_{L j}(t) Y_{1}\left(x_{g i}\right)$

and

- Torsional movement equation

$M_{t} \ddot{q_{t 1}}(t)+C_{t} \dot{q_{t 1}}(t)+K_{t} q_{t 1}(t)=P_{t}(t)$

$$
\begin{aligned}
& M_{t}=1, C_{t}=0, K_{t}=\frac{G K}{\rho I_{0}}\left(\frac{\pi}{l}\right)^{2}+\sum_{i=1}^{N} K_{\psi} \phi_{1}^{2}\left(x_{i}\right) \\
& \text { Where } \\
& P_{t}(t)=\sum_{j=1}^{4}\left(G_{L j}(t) e_{1}-G_{v j}(t) e_{2}\right) \phi_{1}\left(x_{g i}\right) .
\end{aligned}
$$

In order to consistent with rail vertical, lateral and torsional vibration matrix differential equation, the rail vibration system is mathematically written as (19).

$$
M \ddot{q_{1}}(t)+C \dot{q_{1}}(t)+K q_{1}(t)=P(t)
$$

Where $\mathrm{M}, \mathrm{C}$ and $\mathrm{K}$ represent mass matrix, damping matrix and stiffness matrix of the rail vertical, lateral and torsional vibration system respectively $q_{1}(t), \dot{q}_{1}(t)$ and $\ddot{q}_{1}(t)$ are rail displacement vector, velocity vector and acceleration vector, respectively, and $\mathrm{P}$ is the load vector [10].

$$
\begin{gathered}
M=\left[\begin{array}{lll}
1 & 0 & 0 \\
0 & 1 & 0 \\
0 & 0 & 1
\end{array}\right], C=\left[\begin{array}{ccc}
C_{z} & 0 & C_{z y} \\
0 & C_{y} & 0 \\
0 & 0 & C_{t}
\end{array}\right], K=\left[\begin{array}{ccc}
K_{z} & 0 & K_{z y} \\
0 & K_{y} & 0 \\
0 & 0 & K_{t}
\end{array}\right], \\
\ddot{q}_{1}(t)=\left[\begin{array}{l}
\ddot{q}_{z 1}(t) \\
\ddot{q}_{y 1}(t) \\
\ddot{q}_{t 1}(t)
\end{array}\right], \dot{q}_{1}(t)=\left[\begin{array}{l}
\dot{q}_{z 1}(t) \\
\dot{q}_{y 1}(t) \\
\dot{q}_{t 1}(t)
\end{array}\right], q_{1}(t)=\left[\begin{array}{l}
q_{z 1}(t) \\
q_{y 1}(t) \\
q_{t 1}(t)
\end{array}\right] \text { and } P(t)=\left[\begin{array}{c}
P_{z}(t) \\
P_{y}(t) \\
P_{t}(t)
\end{array}\right]
\end{gathered}
$$

\section{THE DifFERENT VARIATION CASES OF THE SYSTEM}

After the mathematical calculation of railway dynamic model, this part focuses on this dynamic model study, where there is the study of each equation of motion and the presentation of different variation cases.

\section{A. Vertical Motion Equation}

This system is a SIMO system (Single Input Multi Output) with two outputs $q_{z 1}(t)$ and $q_{t 1}(t)$.

$\ddot{M_{z}} \ddot{q_{z 1}}(t)+C_{z} \dot{q}_{z 1}(t)+K_{z} q_{z 1}(t)+C_{z y} \ddot{q_{t 1}}(t)+K_{z y} q_{t 1}(t)=P_{z}(t)$

We propose: $C_{z}=\frac{C}{M}, K_{z}=\frac{K}{M}, C_{z y}=\frac{C_{1}}{M}, K_{z y}=\frac{K_{1}}{M}$ and $P_{z}=\frac{F}{M}$.

$\mathrm{C}, \mathrm{C} 1$ are respectively the damping constant of the first and second subsystem, K, K1 are the displacement constant of the first and second subsystem, respectively and $F$ is the result force.

With the superposition method, this system can be divided on two SISO subsystems with $q_{z 1}(t)$ and $q_{t 1}(t)$ output. This method is presented in the following calculation of this paper.

The output $q_{t 1}(t)$ supposed null then the first subsystem like:

$$
\ddot{q_{z 1}}(t)+C_{z} \dot{q_{z 1}}(t)+K_{z} q_{z 1}(t)=P_{z}(t)
$$

Now the second output $q_{z 1}(t)$ supposed null then the second subsystem like:

$C_{z y} \ddot{q}_{t 1}(t)+K_{z y} q_{t 1}(t)=P_{z}(t)$

The final solution of this system is the sum of two outputs $q_{z 1}(t)$ and $q_{t 1}(t)$ since both subsystems are parallel. 
First subsystem (20) is a damped oscillating system with output $q_{z 1}(t)$.

We can take:

$\left\{\begin{array}{c}2 \varepsilon_{z} \omega_{z}=C_{z} \\ \omega_{z}^{2}=K_{z}\end{array}\right.$

$\varepsilon z$ the damping coefficient and $\omega z$ the natural frequency of the system.

Where $H_{a}(p)=\frac{1}{2 \varepsilon_{z} \omega_{z} p+\omega_{z}^{2} p+p^{2}}$

If $\varepsilon z=0$ the system unamortized and $\mathrm{Cz}=0$.

So $\sum_{i=1}^{N} C_{p v}\left(\sin \left(\frac{\pi x_{i}}{l}\right)-\sin \left(\frac{\pi x_{s i}}{l}\right)\right) \sin \left(\frac{\pi x_{i}}{l}\right)=0$

The solution of this system is

$\left\{C_{p v}=0\right.$ or $x_{i}=n l$ or $\left.x_{i}=x_{s i} ; \mathrm{n} \in N\right\}$

Else if $\varepsilon z \neq 0$ the system is a damped oscillating system.

So the study of this part is like the following.

$2 \varepsilon_{z} \omega_{z} p+\omega_{z}^{2} p+p^{2}=0$

$\Delta_{z}=4 \omega_{z}^{2}\left(\varepsilon_{z}^{2}-1\right)$

There are three cases to solve this system.

First case if $\varepsilon_{z}>1$ then $\Delta_{z}<0$ (a periodic regime).

Second case if $\left.\varepsilon_{z} \in\right] 0 \quad 1\left[\right.$ then $\Delta_{z}>0$ (pseudo periodic regime).

Third case if $\varepsilon_{z}=1$ then $\Delta_{z}=0$ (Critic regime).

First, the periodic regime study, $\varepsilon Z>1$.

$F_{z}=C_{p v}^{2} A^{2}-4 K_{p v} D-4 \frac{E I_{y}}{m r}\left(\frac{\pi}{\rho}\right)^{4}>0$

$A=\sum_{i=1}^{N}\left(Z_{1}\left(x_{i}\right)-Z_{1}\left(x_{s i}\right)\right) Z_{1}\left(x_{i}\right)$

Then the solution of this equation is

$\left.A_{z} \in\right]-\infty \quad A_{1}[\cup] A_{2} \quad+\infty[$.

Where $\mathrm{A} 1$ and $\mathrm{A} 2$ are the two roots of this equation.

The solution is written as

$\left.\sum_{i=1}^{N}\left(\sin \left(\frac{\pi x_{i}}{l}\right)-\sin \left(\frac{\pi x_{s i}}{l}\right)\right) \sin \left(\frac{\pi x_{i}}{l}\right) \in\right]-\infty \frac{A_{1} m_{r} l}{2}[\cup] \frac{A_{2} m_{r} l}{2}+\infty[$

Second, the critic regime study. $\varepsilon_{z}=1$ and $F_{z}=0$.

The solution in this case is written as

$$
\sum_{i=1}^{N}\left(\sin \left(\frac{\pi x_{i}}{l}\right)-\sin \left(\frac{\pi x_{s i}}{l}\right)\right) \sin \left(\frac{\pi x_{i}}{l}\right)=\left\{\begin{array}{ll}
\frac{A_{1} m_{r} l}{2} & \frac{A_{2} m_{r} l}{2}
\end{array}\right\}
$$

Third, the pseudo periodic regime study. $\left.\varepsilon_{z} \in\right] 0 \quad 1[$.

In this case the solution is written as

$$
\left.\sum_{i=1}^{N}\left(\sin \left(\frac{\pi x_{i}}{l}\right)-\sin \left(\frac{\pi x_{s i}}{l}\right)\right) \sin \left(\frac{\pi x_{i}}{l}\right) \in\right] 0 \quad \frac{A_{2} m_{r} l}{2}[
$$

If there is no wagon on the rail: $X_{g i}=0$ and $P_{z}=0$. So qz1 $(t)=0$ then it's the case where $t<0$.

Now if there are no sleepers: $X_{s i}=0$ and

$$
\sin \left(\frac{\pi x_{s i}}{l}\right)=0
$$

$\sum_{\text {then }}^{N}\left(\sin \left(\frac{\pi x_{i}}{l}\right)-\sin \left(\frac{\pi x_{s i}}{l}\right)\right) \sin \left(\frac{\pi x_{i}}{l}\right)=\sum_{i=1}^{N}\left(\sin \left(\frac{\pi x_{i}}{l}\right)\right)^{2}$

Finally the general solution of the system is:

$$
\begin{cases}\text { a periodic regime: } & \left.\sum_{i=1}^{N}\left(\sin \left(\frac{\pi x_{i}}{l}\right)\right)^{2} \in\right]-\infty \frac{A_{1} m_{r} l}{2}[\cup] \frac{A_{2} m_{r} l}{2}+\infty[ \\ \text { Critic regime: } & \sum_{i=1}^{N}\left(\sin \left(\frac{\pi x_{i}}{l}\right)\right)^{2}=\left\{\frac{A_{1} m_{r} l}{2} \frac{A_{2} m_{r} l}{2}\right\} \\ \text { Pseudo periodic regime: } & \left.\sum_{i=1}^{N}\left(\sin \left(\frac{\pi x_{i}}{l}\right)\right)^{2} \in\right] 0 \frac{A_{2} m_{r} l}{2}[\end{cases}
$$

Second subsystem is a system with uniform motion (constant speed system) so it can be written as a first order system with an output $\mathrm{q}_{\mathrm{t} 1}(\mathrm{t})$.

We can take:

$\left\{\begin{array}{l}C_{z y}=\frac{\tau}{k} \\ K_{z y}=\frac{1}{k}\end{array}\right.$

Where $\tau$ is the time constant and $\mathrm{k}$ is the static gain. The equation (21) can be written like (29) and the transfer function of this system is written like (30).

$$
\begin{aligned}
& \frac{\tau}{k} \dot{q}_{t 1}(t)+\frac{1}{k} q_{t 1}(t)=P_{z r}(t) \\
& H_{b}=\frac{q_{t 1}(p)}{P_{z r}(p)}=\frac{k}{p \tau+1}
\end{aligned}
$$

The general transfer function vertical motion equation is the sum of (23) and (30):

$$
H_{z}(p)=H_{a}(p)+H_{b}(p)
$$

\section{B. Lateral Motion Equation}

Apply the same principle of the calculation in the previous part for the vertical motion equation but in this case a SISO (Simple Input Simple Output) system. 
$M_{y} \ddot{q}_{y 1}(t)+C_{y} \dot{q}_{y 1}(t)+K_{y} q_{y 1}(t)=P_{y}(t)$

The general solutions of the system are:

$$
\begin{aligned}
& \text { a periodic regime: } \left.\quad \sum_{i=1}^{N}\left(\sin \left(\frac{\pi x_{i}}{l}\right)\right)^{2} \in\right]-\infty \frac{B_{1} m_{r} l}{2}[\cup] \frac{B_{2} m_{r} l}{2}+\infty[
\end{aligned}
$$

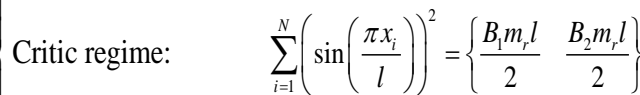

$$
\begin{aligned}
& \text { Pseudo periodique regime: } \left.\sum_{i=1}^{N}\left(\sin \left(\frac{\pi x_{i}}{l}\right)\right)^{2} \in\right] 0 \frac{B_{2} m_{r} l}{2}[
\end{aligned}
$$

\section{Torsional Motion Equation}

In this part also select the same principle as the previous ones. This subsystem is a SISO system.

$$
M_{t} \ddot{q_{t 1}}(t)+C_{t} \dot{q}_{t 1}(t)+K_{t} q_{t 1}(t)=P_{t}(t)
$$

Or $\mathrm{Ct}=0$ so $\varepsilon_{t}=0$ always, in this case the general system is unamortized.

This part presented the detailed study of general system which is divided into three essential parts: the lateral, vertical and torsional motion equations. There is a resolution for each part of nonlinear system based on accurate mathematical calculation in different regimes.

\section{FAult Detection}

After the system study this section focuses on the quantitative diagnosis which contains many mandatory steps to have good results. The error detection this step should make it possible to decide whether the system is in a state of normal running or not, defect location step where the fault elements defined and the decision making step where the incorrect operation of the system being found so the decision how to keep the desired performance of the system under surveillance. The diagnosis can be generated using different methods, a few methods have studied and shown in [16]-[20].

This work deals with one of the finest methods for generating residuals it's the Luenberger Observer (LO) [21]. To prove the efficiency and accuracy of this method, analysis was performed and a result was presented on the following of this paper.

Suppose the linear system affected by a sensor fault, an actuator fault and a measurement noise following:

$$
\left\{\begin{array}{l}
\dot{x}(t)=A x(t)+B u(t)+F_{x} f(\mathrm{t})+\mathrm{D}_{x} d(t) \\
y(t)=C x(t)+F_{y} f(t)
\end{array}\right.
$$

A dynamic observer of this model given by:

$$
\left\{\begin{array}{l}
\dot{\hat{x}}(t)=A \hat{x}(t)+B u(t)+\mathrm{L}(\mathrm{y}-\hat{\mathrm{y}}) \\
\hat{\mathrm{y}}(t)=C \hat{x}(t)
\end{array}\right.
$$

Where, $\mathrm{L}$ is the Observer gain.

The error is: $e_{x}(t)=x(t)-\hat{x}(t) \Leftrightarrow \dot{e}_{x}(t)=\dot{x}(t)-\dot{\hat{x}}(t)$

$$
\begin{aligned}
\dot{e}_{x}(t) & =A x(t)+B u(t)+F_{x} f(t)+D_{x} d(t)-A \hat{x}(t)-B u(t)-L(y(t)-\hat{\mathrm{y}}(\mathrm{t})) \\
& =A x(t)+B u(t)+\mathrm{F}_{x} f(t)+D_{x} d(t)-A \hat{x}(t)-B u(t)-L(y(t)-C \hat{x}(t)) \\
& =A x(t)-A \hat{x}(t)-L(y(t)-C \hat{x}(t))+\mathrm{F}_{x} f(t)+D_{x} d(t) \\
& =A x(t)-L C x(t)-\mathrm{LF}_{y} f(t)+\mathrm{F}_{x} f(t)+D_{x}(t) \mathrm{d}(\mathrm{t})-A \hat{x}(t)+L C \hat{x}(t) \\
& =(A-L C)(x(\mathrm{t})-\hat{x}(t))-\mathrm{LF}_{y} f(t)+\mathrm{F}_{x} f(t)+D_{x}(t) \mathrm{d}(\mathrm{t}) \\
& =(A-L C) e_{x}(t)+\left(F_{x}-L F_{y}\right) f(t)+D_{x}(t) \mathrm{d}(\mathrm{t}) \\
\dot{e}_{x}(t) & =(A-L C) e_{x}(t)+\left(F_{x}-L F_{y}\right) f(t)+D_{x}(t) \mathrm{d}(\mathrm{t})
\end{aligned}
$$

In the absence of default $(\mathrm{f}(\mathrm{t})=0)$ and neglecting the effect of the unknown inputs $(\mathrm{d}(\mathrm{t})=0)$, the estimation error becomes $\dot{e}_{x}(t)=(A-L C) e_{x}(t)$. The objective is $\lim _{t \rightarrow+\infty} e_{x}(t)=0$, this is the case by calculating $\mathrm{L}$.

The dynamic of the state estimation is the residue of the system:

$$
r(t)=y(t)-\hat{y}(t)=C \tilde{x}(t)
$$

As followed from Fig. 1 shown below, the principle of the generation of residues based on the observer.

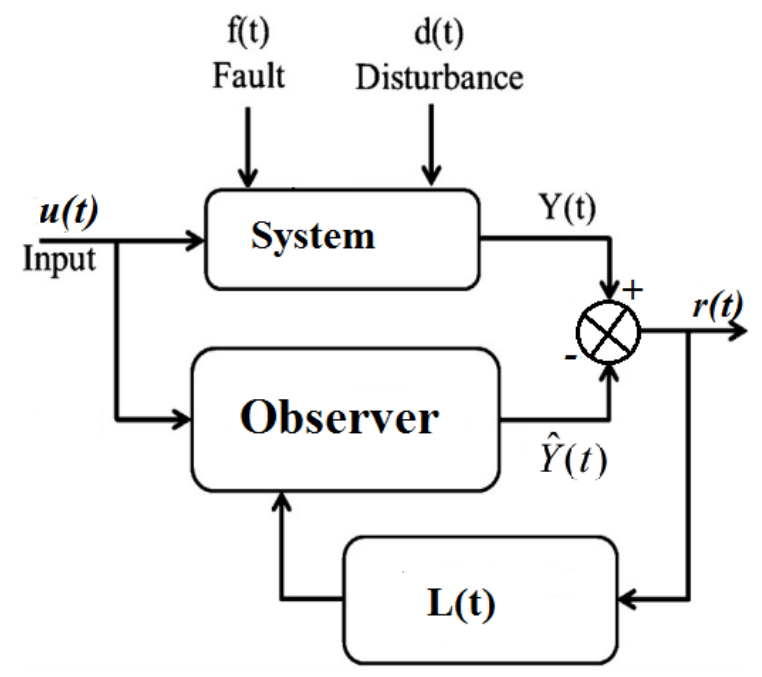

Fig. 1. The principle of the generation of residues based on the observer.

The knowledge that the system contains a defect or not the residue value must be determined, the general conditions of residue presented in (34).

$\left\{\begin{array}{l}r(t) \neq 0 \text { if the default } f(t) \neq 0 \\ r(t)=0 \text { if the default } f(t)=0\end{array}\right.$

\section{APPLICATIONS AND SIMULATIONS}

\section{A. The Global Model}

This part presented the model and study simulations based on the MATLAB program.

Fig. 2 shows the three global outputs of the railway system model, vertical, lateral and torsional displacement. 
The first output is the vertical movement; it is a damped system as Fig. 2(a) shows. It is amortized signal with maximum value in the begin movement equal $120 \mathrm{~mm}$, with damping value $\mathrm{C}=0.0713$ and stiffness value $\mathrm{K}=1.1891$.

The second output is the lateral movement it's a damped system also as Fig. 2(b) shows, but it is more frequency than the first. It is amortized signal with maximum value in the begin movement equal $10 \mathrm{~mm}$, with damping value $\mathrm{C}=0.6454$ and stiffness value $\mathrm{K}=25.8164$.

The third output is the torsional movement, it is unamortized system as Fig. 2(c) shows, but more frequency than the other outputs. It's unamortized signal with maximum value of movement equal $100 \mathrm{~mm}$, with damping value $\mathrm{C}=0$ and stiffness value $\mathrm{K}=173.7861$.

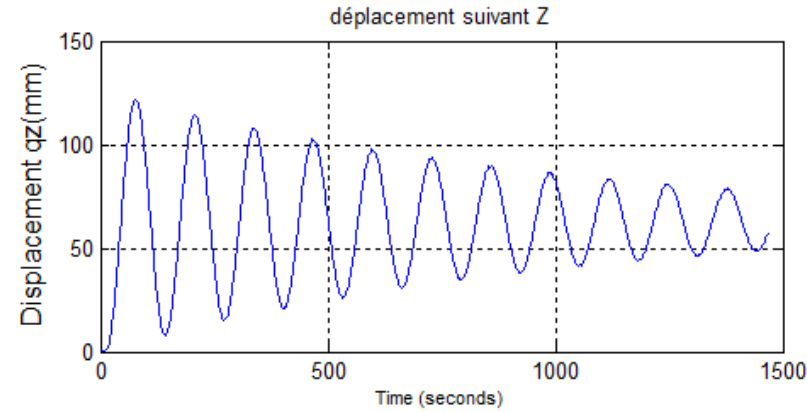

a) The vertical displacement of the rail.

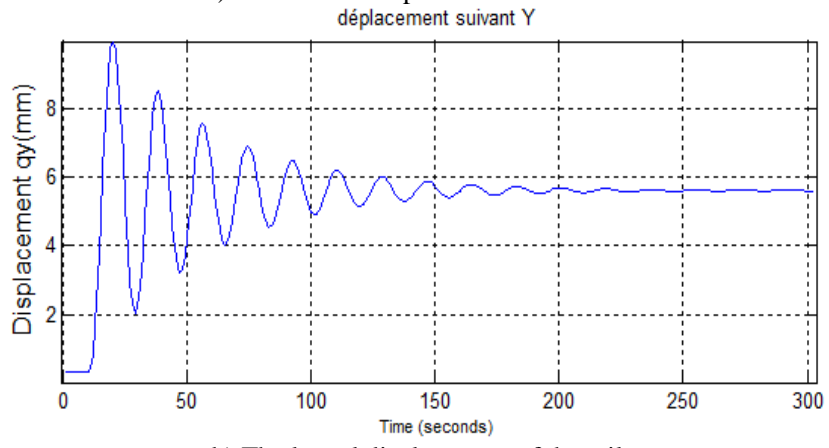

b) The lateral displacement of the rail.

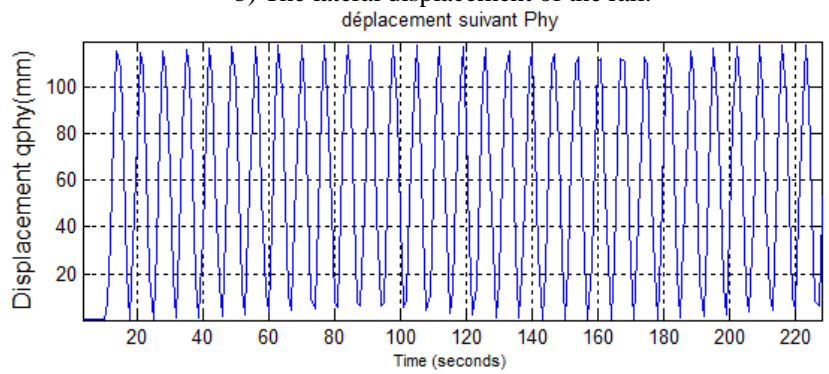

c) The torsional displacement of the rail.

Fig. 2. The railway model system.

\section{B. The Model Study}

Some examples of numerical results in a few positions on the rail are studied such cases are depicted in the following figures:

- First case is the initial position of the system $(X=0)$.
Damping coefficients are $\varepsilon_{z}=0, \varepsilon_{y}=0$ and ${ }^{\varepsilon_{t}}=0$. The natural frequency are $W z=0.0043, W y=0.0047$ and $W t=0.2602$.

The Transfer function $\mathrm{Hz}$ (vertical movement), Hy (Lateral movement) and $\mathrm{Ht}$ (Torsional movement) respectively like these:

$H z(p)=\frac{1}{p^{2}+1.83310^{-5}}, H y(p)=\frac{1}{p^{2}+2.210^{-5}}$

and $H t(p)=\frac{1}{p^{2}+0.067}$

Fig. 3 illustrates the transfer functions of three motions directions. Like the simulations present they are unamortized then the system is in a Critic regime.
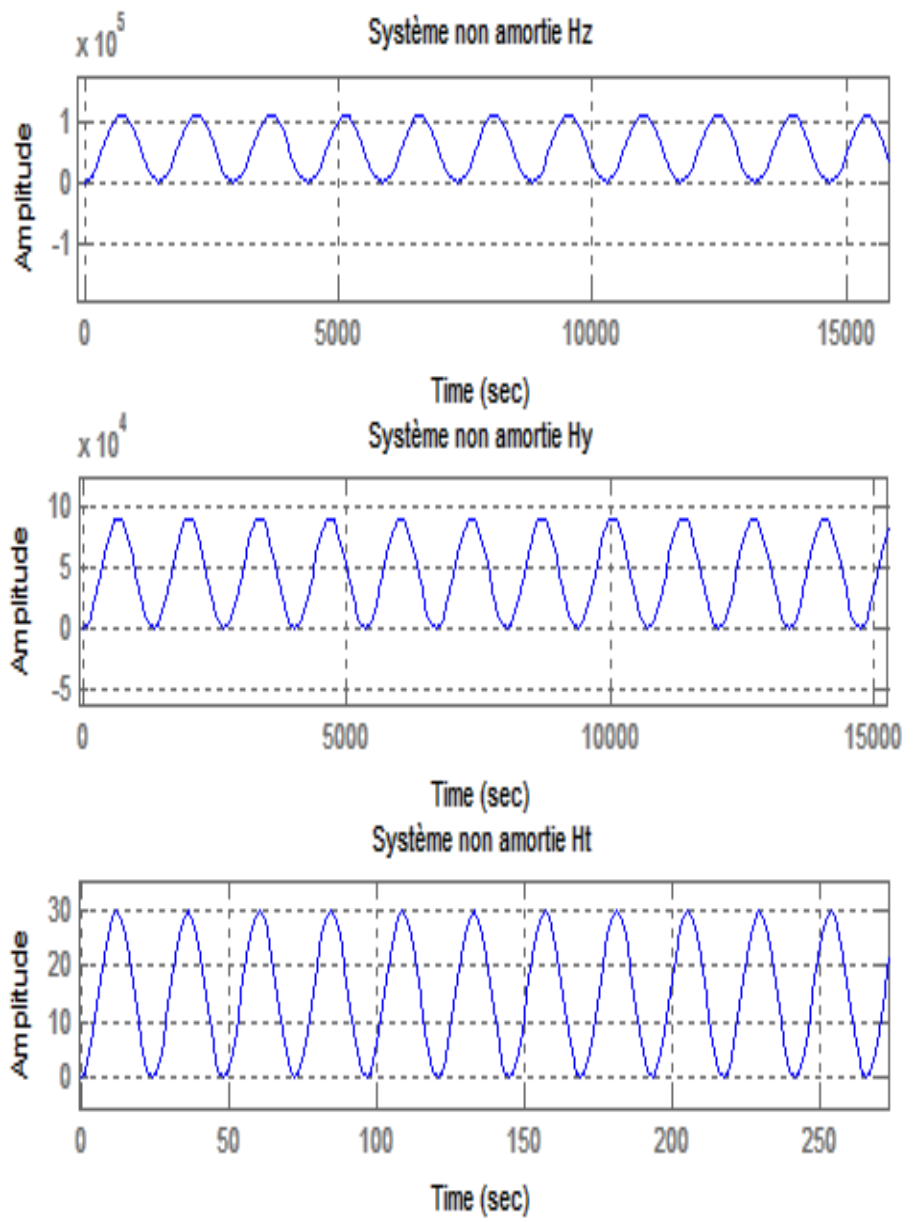

Fig. 3. Transfer function of the system in the first case.

- Second case is the seventh position for the system $(\mathrm{X}=7)$.

Damping coefficients are $\varepsilon_{z}=0.539$ and $\varepsilon_{y}=0.305$. The natural frequency are $W z=1.295, W y=0.915$. The Transfer function $\mathrm{Hz}$ and $\mathrm{Hy}$, respectively like these: 
$H z(p)=H a(p)+H b(p) \quad$ where $\left\{\begin{array}{l}H a(p)=\frac{1}{p^{2}+1.398 p+1.678} \\ H b(p)=\frac{1}{0.833 p+1.678}\end{array}\right.$

$H y(p)=\frac{1}{p^{2}+0.559 p+0.838}$

The simulation of these transfer functions illustrated in Fig. 4. Like these simulations present they are in a pseudo periodic regime then the system is amortized.
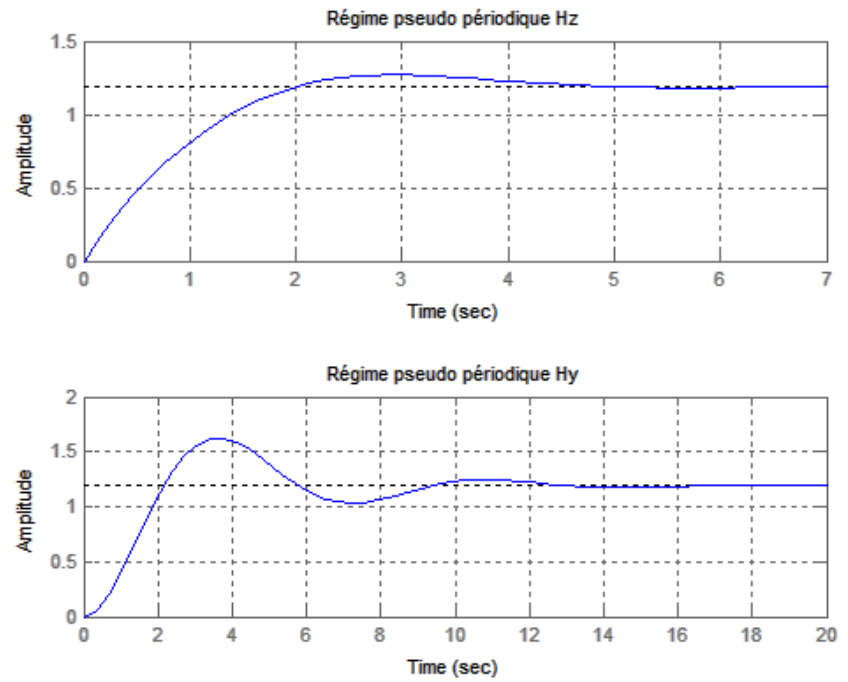

Fig. 4. Transfer function of the system in the second case.
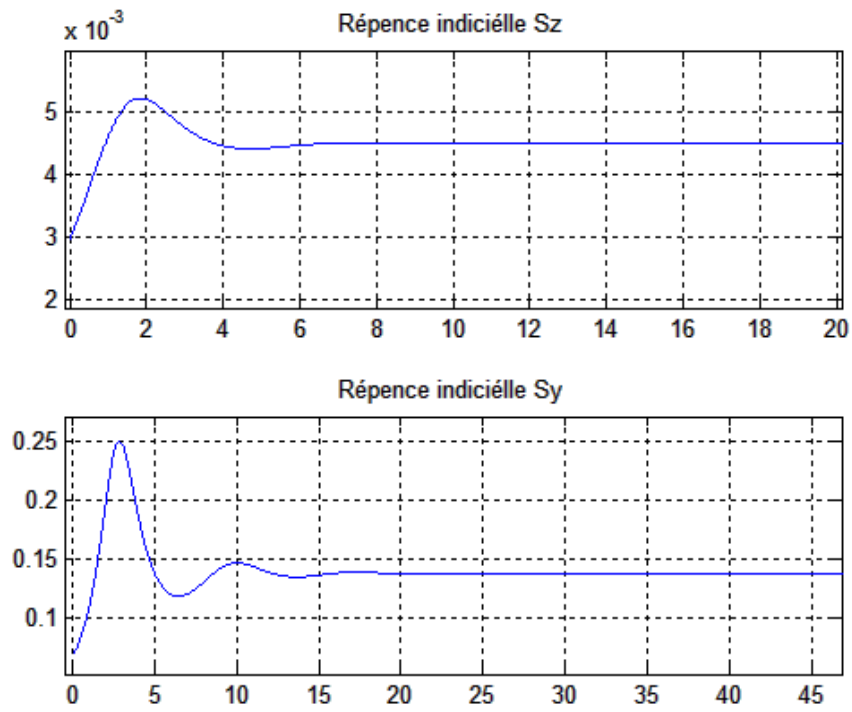

Fig. 5. The Step response of the system (Sz and Sy) in the second case.

The step response of this system with step unit function input is presented in Fig. 5.

- Third case is the final position for the system $(X=30)$.
Damping coefficients in this case are $\varepsilon_{y}=2.2045$ and $\varepsilon_{z}=3.8971$. The natural frequency are $W z=9.3531$, $W y=6.6136$.

The Transfer function $\mathrm{Hz}$ and $\mathrm{Hy}$ :

$H z(p)=H a(p)+H b(p)$ where $\left\{\begin{array}{l}H a(p)=\frac{1}{p^{2}+72.9 p+87.48} \\ H b(p)=\frac{1}{0.83 p+87.48}\end{array}\right.$

$H y(p)=\frac{1}{p^{2}+29.16 p+43.74}$

The simulations of these transfer functions are given in Fig. 6. From this figure it can be see that the global system become a first order system and the simulation are in a periodic regime.
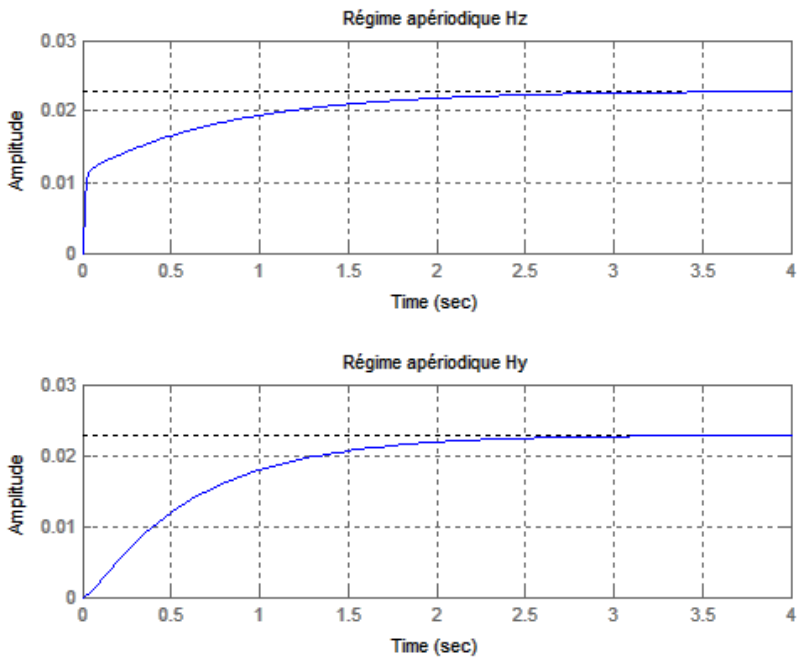

Fig. 6. Transfer function of the system in the third case.
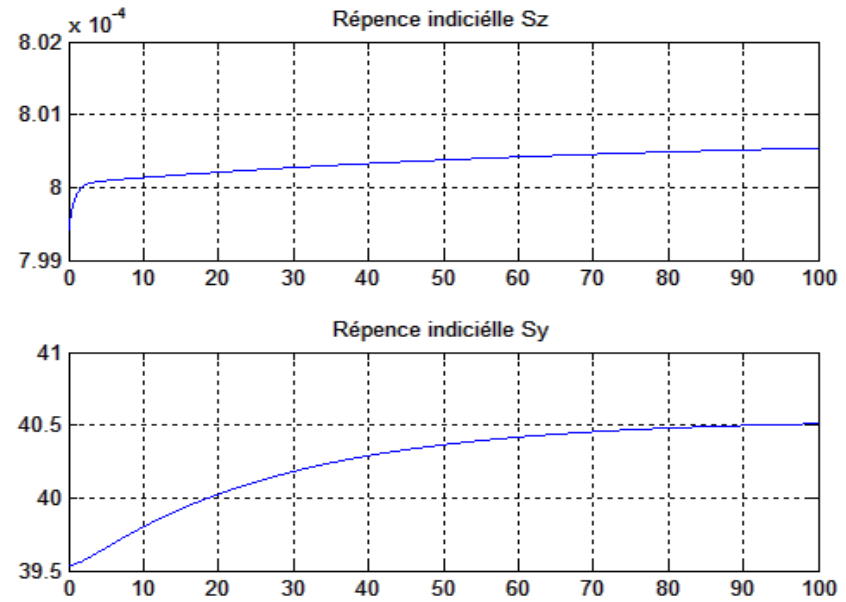

Fig. 7. The step response of the system $(\mathrm{Sz}, \mathrm{St})$ in the third case.

With the input of the system is step unit function, the step response being found in Fig. 7. 


\section{The Fault Detection}

To calculate and isolate the system fault, first it must calculate the global system model which is done in the following. First there is a model calculation then an application for the LO, the numerical results are presented and the simulations are done.

The general model (19) demonstrate that the railway system have three defaults, one default on each side of vibration $\mathrm{f}(\mathrm{t})$. The simulation in this paper with no disturbance $(\mathrm{d}(\mathrm{t})=0)$.

To complete this work it must linearize the system to apply the luenberger observer. So after the linearization, the railway system described by the following state representation:

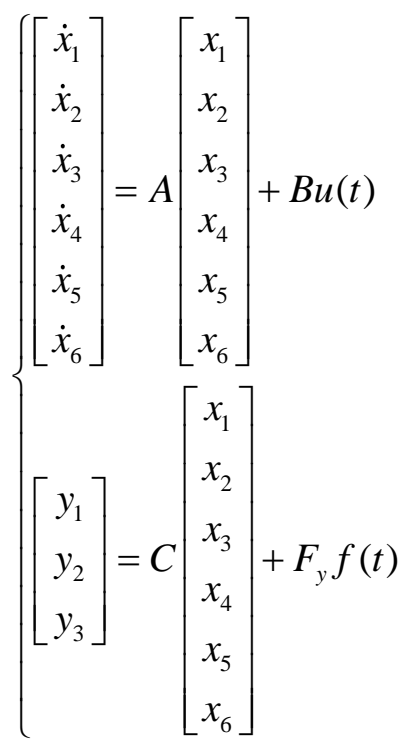

Matrices of the state are:

$A=\left[\begin{array}{cccccc}0 & 1 & 0 & 0 & 0 & 0 \\ -K_{z} & -C_{z} & 0 & 0 & -K_{z y} & -C_{z y} \\ 0 & 0 & 0 & 1 & 0 & 0 \\ 0 & 0 & -K_{y} & -C_{y} & 0 & 0 \\ 0 & 0 & 0 & 0 & 0 & 1 \\ 0 & 0 & 0 & 0 & -K_{t} & 0\end{array}\right], B=\left[\begin{array}{ccc}0 & 0 & 0 \\ 1 & 0 & 0 \\ 0 & 0 & 0 \\ 0 & 1 & 0 \\ 0 & 0 & 0 \\ 0 & 0 & 1\end{array}\right]$

$C=\left[\begin{array}{llllll}1 & 0 & 0 & 0 & 0 & 0 \\ 0 & 0 & 1 & 0 & 0 & 0 \\ 0 & 0 & 0 & 0 & 1 & 0\end{array}\right], F_{y}=\left[\begin{array}{lll}1 & 0 & 0 \\ 0 & 1 & 0 \\ 0 & 0 & 1\end{array}\right], f(t)=\left[\begin{array}{l}f_{1}(t) \\ f_{2}(t) \\ f_{3}(t)\end{array}\right]$

and $u(t)=\left[\begin{array}{l}u_{1}(t) \\ u_{2}(t) \\ u_{3}(t)\end{array}\right]$

In the case $\mathrm{N}=20$ the numerical values are founded as follow:

$$
\begin{aligned}
& K_{z}=0.4951, C_{z}=0.0297, K_{z y}=0.6940, C_{z y}=0.0416, \\
& K_{y}=25.8164, C_{y}=0.6454, K_{t}=173.7861 .
\end{aligned}
$$

The state representation of system in the expanded form:

$$
\begin{aligned}
& \left\{\begin{array}{l}
{\left[\begin{array}{l}
\dot{x}_{1} \\
\dot{x}_{2} \\
\dot{x}_{3} \\
\dot{x}_{4} \\
\dot{x}_{5} \\
\dot{x}_{6}
\end{array}\right]=A_{1}\left[\begin{array}{l}
x_{1} \\
x_{2} \\
x_{3} \\
x_{4} \\
x_{5} \\
x_{6}
\end{array}\right]+B_{1} \mathrm{U}(t)} \\
{\left[\begin{array}{l}
y_{1} \\
y_{2} \\
y_{3}
\end{array}\right]=C_{1}\left[\begin{array}{l}
x_{1} \\
x_{2} \\
x_{3} \\
x_{4} \\
x_{5} \\
x_{6}
\end{array}\right]+D_{1} U(t)}
\end{array}\right. \\
& A_{1}=A, B_{1}=\left[\begin{array}{llllll}
0 & 0 & 0 & 0 & 0 & 0 \\
1 & 0 & 0 & 0 & 0 & 0 \\
0 & 0 & 0 & 0 & 0 & 0 \\
0 & 1 & 0 & 0 & 0 & 0 \\
0 & 0 & 0 & 0 & 0 & 0 \\
0 & 0 & 1 & 0 & 0 & 0
\end{array}\right], C_{1}=C, \\
& D_{1}=\left[\begin{array}{llllll}
0 & 0 & 0 & 1 & 0 & 0 \\
0 & 0 & 0 & 0 & 1 & 0 \\
0 & 0 & 0 & 0 & 0 & 1
\end{array}\right] \text { and } U=\left[\begin{array}{l}
u_{1}(t) \\
u_{2}(t) \\
u_{3}(t) \\
f_{1}(t) \\
f_{2}(t) \\
f_{3}(t)
\end{array}\right]
\end{aligned}
$$

The actual structure of the $\mathrm{LO}$ is:

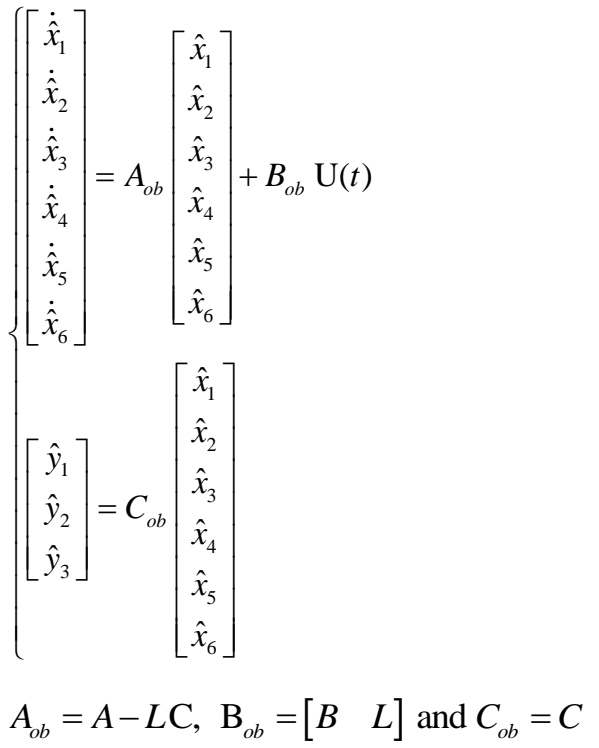


The eigenvalues of $A_{o b}$ are $\lambda_{1}=-5, \lambda_{2}=-9, \lambda_{3}=-6, \lambda_{4}=-5$, $\lambda_{5}=-2$ and $\lambda_{6}=-10$. These eigenvalues gives the gain Matrix L.

$$
\mathrm{L}=\left[\begin{array}{lll}
11.6844 & -1.4550 & -1.0819 \\
19.7897 & -4.1470 & -9.1942 \\
-1.2283 & 6.9358 & -0.7012 \\
-2.4554 & -22.2438 & -2.7048 \\
-1.2962 & -0.8920 & 13.7047 \\
-9.2933 & -4.3545 & -130.8955
\end{array}\right]
$$

Therefore the numerical values of the state matrices for $\mathrm{LO}$ are:

$$
\begin{aligned}
A_{o b} & =\left[\begin{array}{cccccc}
-11.6844 & 1 & 1.4550 & 0 & 1.0819 & 0 \\
-20.2848 & -0.0297 & 4.1470 & 0 & 8.5002 & -0.0416 \\
1.2283 & 0 & -6.9358 & 1 & 0.7012 & 0 \\
2.4554 & 0 & -3.5725 & -0.6454 & 2.7048 & 0 \\
1.2962 & 0 & 0.8920 & 0 & -13.7047 & 1 \\
9.2933 & 0 & 4.3545 & 0 & -42.8907 & 0
\end{array}\right] \\
\mathrm{B}_{o b} & =\left[\begin{array}{lllllll}
0 & 0 & 0 & 11.6844 & -1.4550 & -1.0819 \\
1 & 0 & 0 & 19.7897 & -4.1470 & -9.1942 \\
0 & 0 & 0 & -1.2283 & 6.9358 & -0.7012 \\
0 & 1 & 0 & -2.4554 & -22.2438 & -2.7048 \\
0 & 0 & 0 & -1.2962 & -0.8920 & 13.7047 \\
0 & 0 & 1 & -9.2933 & -4.3545 & -130.8955
\end{array}\right] \\
C_{o b} & =\left[\begin{array}{llllll}
1 & 0 & 0 & 0 & 0 & 0 \\
0 & 0 & 1 & 0 & 0 & 0 \\
0 & 0 & 0 & 0 & 1 & 0
\end{array}\right]
\end{aligned}
$$

The system provides three outputs $q_{z}(t), q_{y}(t)$ and $q_{t}(t)$ are respectively the vertical, lateral and torsional displacement of rail because the vibration. The simulation of the real and estimate outputs of the railway system then the simulation of the residual of each default in the following of the paper finally the conclusion of fault detection part.

In the simulation, the default value, $f(t)=\left[\begin{array}{c}0.5 \\ -0.6 \\ 0.1\end{array}\right]$.

Fig. 8 defines the real and estimate vertical displacements of rail they are respectively $\mathrm{Y} 1 \mathrm{r}$ and $\mathrm{Y} 1 \mathrm{es}$.

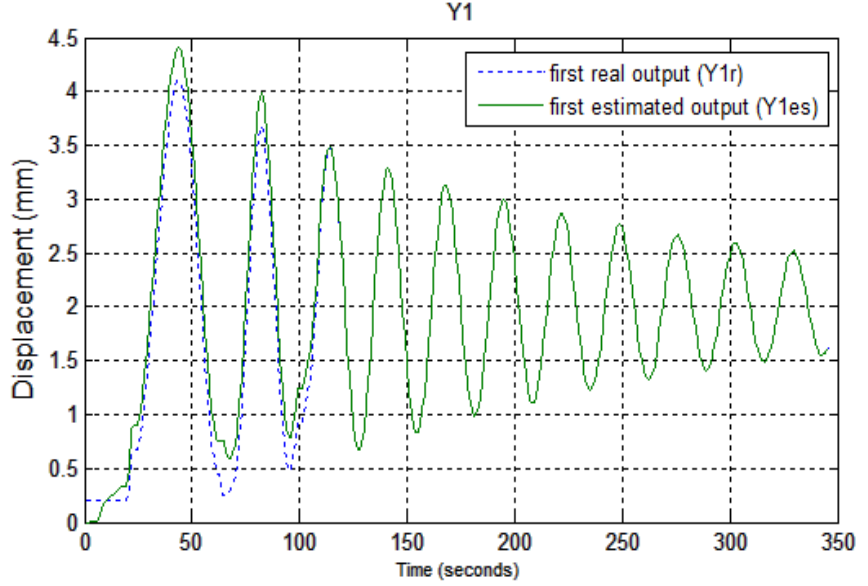

Fig. 8. Real and estimated outputs.

Fig. 9 indicates the real and estimate lateral displacements of rail they are respectively Y2r and Y2es.

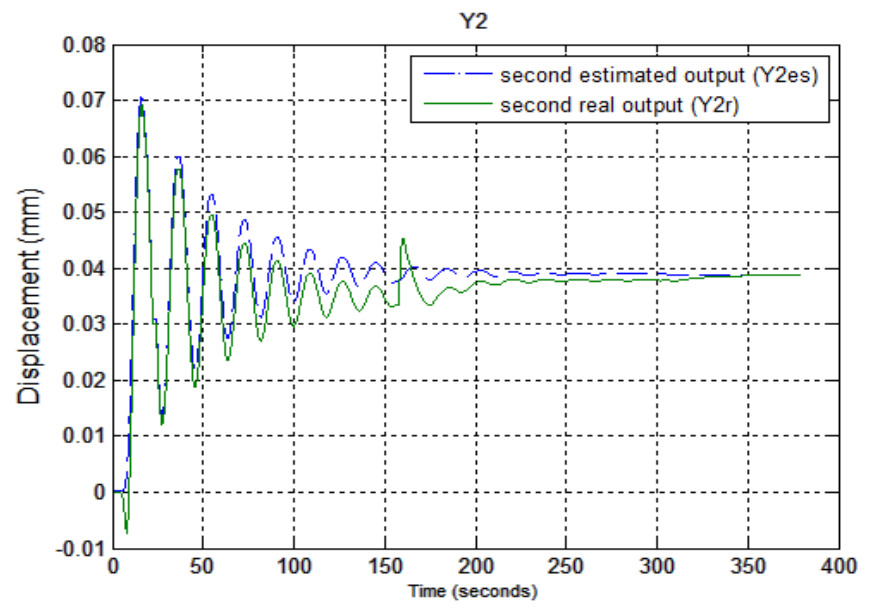

Fig. 9. Real and estimate second outputs.

Fig. 10 depicts the real and estimate torsional displacements of rail they are respectively Y3r and Y3es.

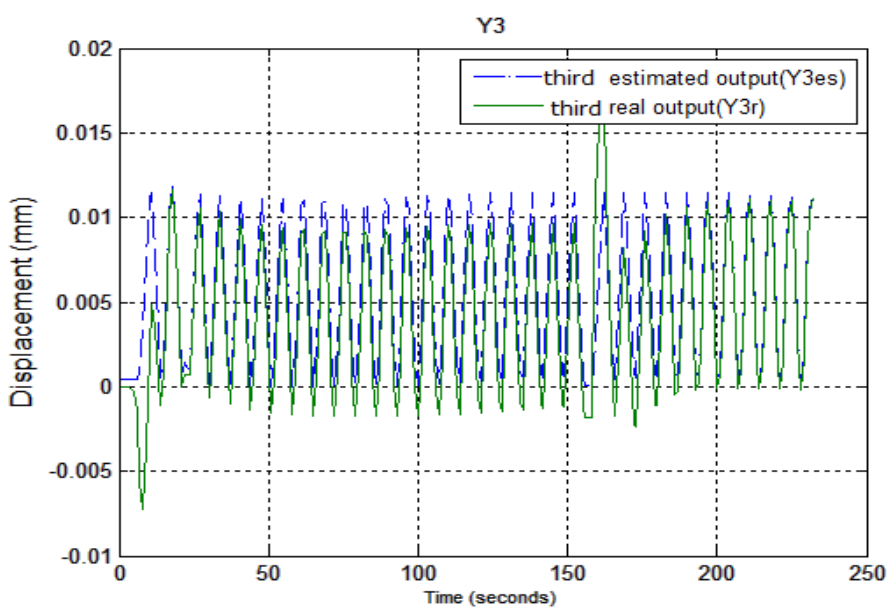

Fig. 10. Real and estimate third outputs. 
This fault case of $f(t)$ gives a residue value to each output of the railway system, these values are described in the simulations of the following figures.

The fault on the first output in this case where:

$f_{1}(t)=\left\{\begin{array}{rr}0.5 & \text { if } \mathrm{t}=500 \mathrm{~s} \\ 0 & \text { elsewhere }\end{array}\right.$

This case is shown in Fig. 11 where the result of the residue $\mathrm{r} 1$ is sensitive to the fault.

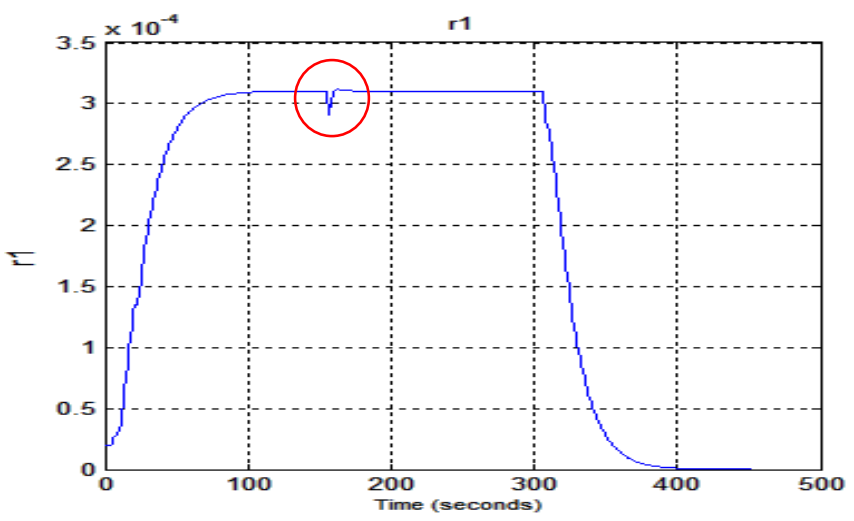

Fig. 11. The residue $r 1$ with default $f_{1}(t)$.

Now the simulation for the effect of the second value $f_{2}(t)$ on the residue $\mathrm{r} 2$ of second output found is shown in Fig. 12.

$f_{2}(t)=\left\{\begin{array}{cl}-0.6 & \mathrm{t}=\{10 \mathrm{~s} \text { and } 301 \mathrm{~s}\} \\ 0 & \text { elsewhere }\end{array}\right.$

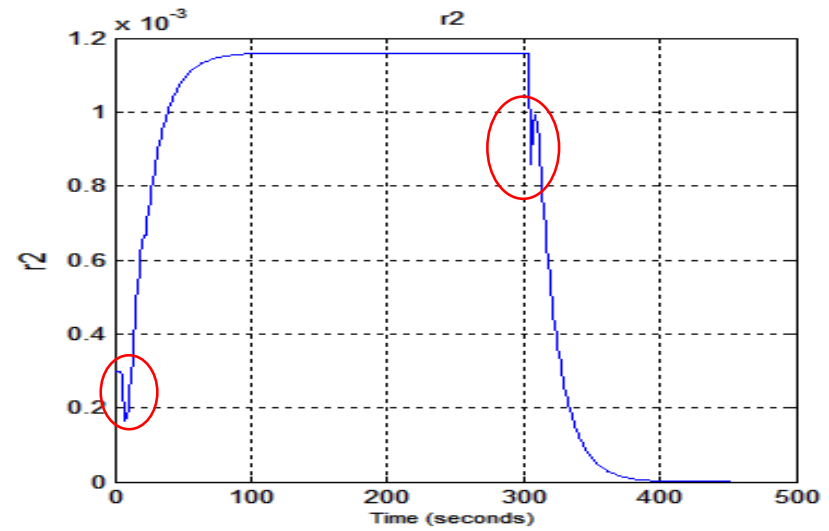

Fig. 12. Residual $\mathrm{r} 2$ with default $\mathrm{f}_{2}(\mathrm{t})$.

Finally, the case of the effect of the third default on third residue $r 3$ of the third output is:

$f_{3}(t)= \begin{cases}0.1 & \mathrm{t}=57 \mathrm{~s} \\ 0 & \text { elsewhere }\end{cases}$

With this value of default, Fig. 13 shows the result of the residual $\mathrm{r} 3$ is sensitive to the fault $f_{3}(t)$.

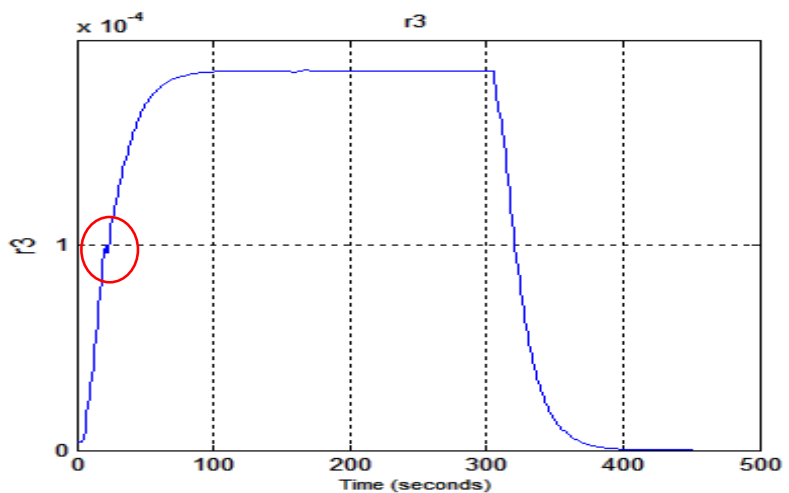

Fig. 13. Residual $r 3$ with fault $\mathrm{f}_{3}(\mathrm{t})$.

\section{VII.CONCLUSION}

Based on the results, it can be concluded that the research into the detection and isolation the default of a railway system has been very successful. A complete model for simulating dynamic interactions between rail and wheels has been presented. An explicit integration algorithm has been employed to numerically solve the equations of motion of the system. After linearization, is studied the linear system and numerical results presented in this work. With the model of railway system we made an embedded system for the robust prediction and diagnosis that can guarantee the security of railway system. A Luenberger Observer (LO) used to generate residuals for detection of faults of a MIMO system.

The future research will concentrate on detection of default based on nonlinear local observer for the railway system and prediction for this default. The future work based on the Local exponential Observer and will apply the results of Sundarapandian research on this type of observer [32], [33].

\section{REFERENCES}

[1] Wanming Zhai, Jianmin Gao, Pengfei Liu and Kaiyun Wang, "Reducing rail side wear on heavy-haul railway curves based on wheel-rail dynamic interaction" Train and Track Research Institute, State Key Laboratory of Traction Power, Southwest Jiaotong University, Chengdu, 610031, People's Republic of China, Receid 31 October 2013; accepted 15 March 2014.

[2] A. Johansson, M. Bask and T. Norlander, "Dynamic Threshold Generators for Robust Fault Detection in linear Systems with Parameter Uncertainty", Automatica. 42 (2006). pp. 1095-1106, 2006.

[3] Soukup J., Skočilas J.and Skočilasová B, "Vertical vibration of the vehicle model with higher degree of Freedom", Sciencedirect. Procedia Engineering 96 ( 2014 ) 435 - 443.

[4] S. Victorovich Makarov, F. Abdulnazarov and O. Anarbekov, "The Development of the Routing Pattern of the Backbone Data Transmission Network for the Automation of the Krasnoyarsk Railway,” International Journal of Advanced Computer Science and Applications (IJACSA),Vol. 7, No. 6, 2016,pp. 107-117.

[5] C.Esveld, Modern Railway Track, MRT-Productions, Zaltbommel, Netherlands, 2001.

[6] S. Zhu, Q. Fu, C. Cai and P.D. Spanos, "Damage evolution and dynamic response of cement asphalt mortar layer of slab track under vehicle dynamic load", Science China Technological Sciences 57 (2014) 18831894.

[7] Zhai, W.M., "Coupling Model of Vertical and Lateral Vehicle/Track Interactions", Vehicle System Dynamics, 26(1996), pp61-79.

[8] S.Zhu and C.Cail, "A nonlinear and fractional derivative viscoelastic model for rail pads in the dynamic analysis of coupled vehicle-slab 
track systems", Journal of Sound and Vibration 335 (2015) 304-320, Elsevier.

[9] N. Gil-Negrete, J. Viñolas and L. Kari, "A simplified methodology to predict the dynamic stiffness of carbon-black filled rubber isolators using a finite element code", Journal of Sound and Vibration 296 (2006) 757-776.

[10] Mingjian Sun, Yan Wang, Xin Zhang, Yipeng Liu, Qiang Wei, Yi Shen and Naizhang Feng, "Feature Selection and Classification Algorithm for Non-destructive Detecting of High-speed Rail Defects Based on Vibration Signals", IEEE, 2014.

[11] Hongyin Yang, Zhijun Chen, Shaofan Li, Hailong Zhang and Jianping Fan, " an integrated coupling element for vehicle-rail-bridge interaction system with a non-uniform continuous bridge", Published by AMSS Press, Wuhan, China. Received 25 June 2013, revision received 20 May 2014.

[12] N. Gil-Negrete, J. Vinolas and L. Kari, “ A Nonlinear, Rubber material model combining fractional order viscoelasticity and amplitude dependent effects", Journal of Applied Mechanics 76 (2009). (011009011009).

[13] K.L. Knothe and S.L. Grassie, "Modeling of railway track and vehicle track interaction at high-frequencies", Vehicle System Dynamics 22 (1993) 209-262.

[14] M.M. Sjöberg and L. Kari, "Non-linear behavior of a rubber isolator system using fractional derivatives", Vehicle System Dynamics 37 (2002) 217-236.

[15] Y. Cai, H. Sun and C. Xu, "Three-dimensional analyses of dynamic responses of track-ground system subjected to a moving train load", 2008, Elsevier.

[16] Naveen Bhargav, Avanish Gupta, Mayank Khirwar, Satypal Yadav and Vinay Sahu, "Automatic Fault Detection of Railway Track System Based on PLC (ADOR TAST)," Naveen Bhargav et al. International Journal of Recent Research Aspects ISSN: 2349-7688, Vol. 3, Issue 1, March 2016, pp. 91-94.

[17] J. Chen, C. Roberts and P. Weston, "Fault detection and diagnosis for railway track circuits using neuro-fuzzy systems," Control Engineering Practice 16 (2008) 585-596.

[18] R. Isermann, "Fault-Diagnosis Systems: An Introduction from Fault Detection to Fault Tolerance" Springer.

[19] Takashi Kojima, Hitoshi Tsunashima and Yoshitaka Marumo, "Real Time Fault Detection of Railway Vehicles and Tracks",The Institution of Engineering and Technology International Conference on Railway Condition Monitoring,2006.

[20] Masato abe, "The Dynamics of Vehicles on Roads and on Tracks Supplement to Vehicle System".

[21] Zhou, B., Li, Z.Y. and Lin, Z. (2012), "Observer based output feedback control of linear systems with multiple input and output delays", in IEEE 51st Annual Conference on Decision and Control (CDC), pp. 23702375.

[22] Hisham A. H. Al-Khazali and Mohamad R. Askari, "Defect Diagnosis in Rotors Systems by Vibrations Data Collectors Using Trending Software", International Journal of Advanced Computer Science and Applications (IJACSA), Vol. 7, No. 5, 2012,pp. 33-43.

[23] Chakkour Saad, Baghouri Mostafa and Hajraoui Abderrahmane, "Performance Analysis of Faults Detection in Wind Turbine Generator Based on High-Resolution Frequency Estimation Methods", International Journal of Advanced Computer Science and Applications (IJACSA),Vol. 5, No. 4, 2014,pp. 139-148.

[24] Mohamed Farhat, Yasser Gritli and Mohamed Benrejeb,"Fast-ICA for Mechanical Fault Detection and Identification in Electromechanical Systems for Wind Turbine Applications", International Journal of Advanced Computer Science and Applications (IJACSA),Vol. 8, No. 7, 2017,pp. 431-439.

[25] N. Baydar, A. Ball, "Detection of gear failures via vibration and acoustic signals using wavelet transform", Mechanical Systems and Signal Processing, vol. 17, no. 4, pp. 787-804, 2003.

[26] Z. Xing yuan, T. Ji Liang, and D. De cun, "Separation of the Train Vibration Signal Based on Improved Fast-ICA", IEEE Computer Society, Fourth International Conference on Intelligent Computation Technology and Automation, Washington, Vol. 1, pp. 912-914, 2011.

[27] C.Roberts, H.P.B.Dassanayake, N.Lehrasab and C.J.Goodman, "Distributed quantitative and qualitative fault diagnosis: railway junction case study", Elsevier, Control Engineering Practice, Volume 10, Issue 4, April 2002, Pages 419-429.

[28] W. Sammouri, E. Côme and L. Oukhellou, "Temporal association rule mining for the preventive diagnosis of onboard subsystems within floating train data framework", 5th International IEEE Conference on Intelligent Transportation Systems (ITSC), 2012.

[29] Claes Johnson, "Numerical Solution of Partial Differential Equations by the Finite Element Method", Dover Publication,INC. Mineola,New York.

[30] U.Fingberg, "A model of wheel-rail squealing noise", Elsevier, Journal of Sound and Vibration, Volume 143, Issue 3, 22 December 1990, Pages 365-377.

[31] Ahmed A.Shabana, Khaled E.Zaazaa, José L.Escalona and Jalil R.Sany, "Development of elastic force model for wheel/rail contact problems", Elsevier, Journal of Sound and Vibration, Volume 269, Issues 1-2, 6 January 2004, Pages 295-325.

[32] Sundarapandian Vaidyanathan, "Local Observer Design For Nonlinear Control Systems Around Equilibria", International Journal of Computer Science, Engineering and Applications (IJCSEA) Vol.2, No.2, April 2012.

[33] Sundarapandian Vaidyanathan, "Local Observer Design for Nonlinear Systems", Mathematical and Computer Modelling 35 (2002) 25-36. 